\title{
Light/Electron Microscopy Correlative Workflow Involving Leica Stage Overview and Thermo Fisher/FEI MAPS Platforms Facilitate Neuronal Identification
}

\author{
Christine A. Brantner ${ }^{1}$, Yigal Lilach ${ }^{1}$, Cheryl Clarkson ${ }^{1}$, Pratik Mistry ${ }^{2}$, Liz Matsiyevskiy ${ }^{2}$, Beverly \\ Karpinski $^{3}$, and Anastas Popratiloff ${ }^{1}$ \\ 1. George Washington University Nanofabrication and Imaging Center, Washington, DC. \\ 2. George Washington University, School of Engineering and Applied Sciences, Department of \\ Biomedical Engineering, Washington, DC. \\ 3. George Washington University, School of Medicine, Department of Anatomy, Washington, DC.
}

Combing light microscopy (LM) and electron microscopy (EM) in Correlative Light Electron Microscopy workflows (re)capture it's place among state of the art imaging technologies available on both light and electron microscopes. Each of the imaging modalities presents advantages and limitations, whereas with complementary approaches, one expects to overcome limitations and amplify advantages. Light microscopy (LM) (either confocal or wide field) is broadly used in biomedical research because of the convenience and less common because of the analytical capabilities offered by the photon. A wide variety of cellular labels are available along with reporting fluorescence proteins whose expression can be genetically promoted by wide variety of genes. Moreover, LM imaging can be employed in living specimens where it can provide time-resolved data sets with meaningful information on biological time scale. Although over the past decade a significant effort has been made to overcome the diffraction limitation imposed by the photon, LM is still a diffraction-limited technique. Scanning electron Microscopy (SEM) naturally provides biologically relevant resolution. Routinely, images with a resolution of a few nanometers can be obtained on many of the modern SEMs. This is the kind of resolution required for reliable characterization of cellular and subcellular morphology of normal and abnormal neuronal circuitry. The latter approach offers full spectrum of morphological characterization, but the cells and tissues need to be strongly fixed. Here we provide a simple workflow, using different LM imaging modalities to facilitate the identification of an individual neuron in trigeminal explant cell culture followed by SEM. The aim of the study is to evaluate the axonal growth and the morphology of growth cone.

Trigeminal ganglia were removed from 9-day-old mouse embryos by micro-dissection and plated on either coated, gridded glass coverslips or in house made silicon chips. The cultures were grown in incubator for 24 hours, followed by fixation with glutaraldehyde and paraformaldehyde. Immunolabeling was performed for neuron specific tubulin (Tuj1) and actin filament was labeled with phaloidin conjugated to Alexa Fluor. After fixation, the cells were processed for critical point drying and sputter coated with $1 \mathrm{~nm}$ of iridium.

A PMMA grid was printed on silicon chips to streamline the identification of neurons in both LM and EM. The printed grid was created by spin coating a silicon chip with $500 \mathrm{~nm}$ of PMMA (two repeats of spin coating of PMMA A4 at $2500 \mathrm{rpm}$ ) then baking on a hotplate for 2 minutes at $180^{\circ} \mathrm{C}$. The PMMA is exposed to an electron beam using a Raith Voyager electron beam lithography tool, using a $50 \mathrm{kV}$ beam. The beam was set to use a high current mode of $11.6 \mathrm{nA}$ and a step size of $50 \mathrm{~nm}$. The electron dose used was $20,000 \mu \mathrm{C} / \mathrm{cm}^{2}$, which resulted in the PMMA behaving as a negative resist. The unexposed resist is removed by acetone. Alternatively, commercially available grid-etched glass slides were used with inverted microscope for similar procedure. 
As a first step, the entire area of both sample types including the grid was captured on a Leica SP8 confocal system equipped with Leica DFC 365FX EM CCD camera (1392x1040px). Leica HC IRAPO $25 \mathrm{x} / 1.00$ water immersion objective with motorized collar was used for all the imaging. The image data was integrated via newly released "Stage Overview" module. Since the purpose of this phase of the experiment was to create interactive map of the sample, the camera image was binned $2 \times 2$ to speed the acquisition with reasonable resolution of a cell body. The resulting image then can be utilized to navigate the field of view for high-resolution imaging. As a next layer, a high-resolution tile set of images was obtained by scanning the sample with the same objective lens, but using confocal laser scanning imaging modality at Nyquist pixel size (optimal resolution). We used super continuum white laser for excitation, which is reflected to the sample with combination of notch filters and acoustooptical beam splitter. The fluorescence signal was detected with hybrid detectors using photon counting. Thus a wide dynamic range data was achieved.

After processing for SEM, the coverslips were placed in FEI/ThermoFisher Teneo Field Emission Scanning Electron Microscope. LM images from the Leica system were then imported directly into the FEI/ThermoFisher MAPS software and aligned to the sample corners. Low-resolution tile scan of the entire sample was first taken using Everhart-Thornley detector for secondary electrons and finely aligned to the low resolution LM images. Then high-resolution secondary electron SEM images was stitched from areas of specific interests such as large clusters of neurons. Cells were identified as neurons base upon Tuj1 labeling in the LM images, whereas negative cells were excluded from further analyses. This approach allowed us to perform precise evaluation of the growth cone morphology and axonal branching [1].

References:

[1] The authors acknowledge funding from NICHHD DC-IDDRC (U54HD090257) and Pathology, Developmental Origins, and Prevention of Pediatric Dysphagia (P01HD083157).

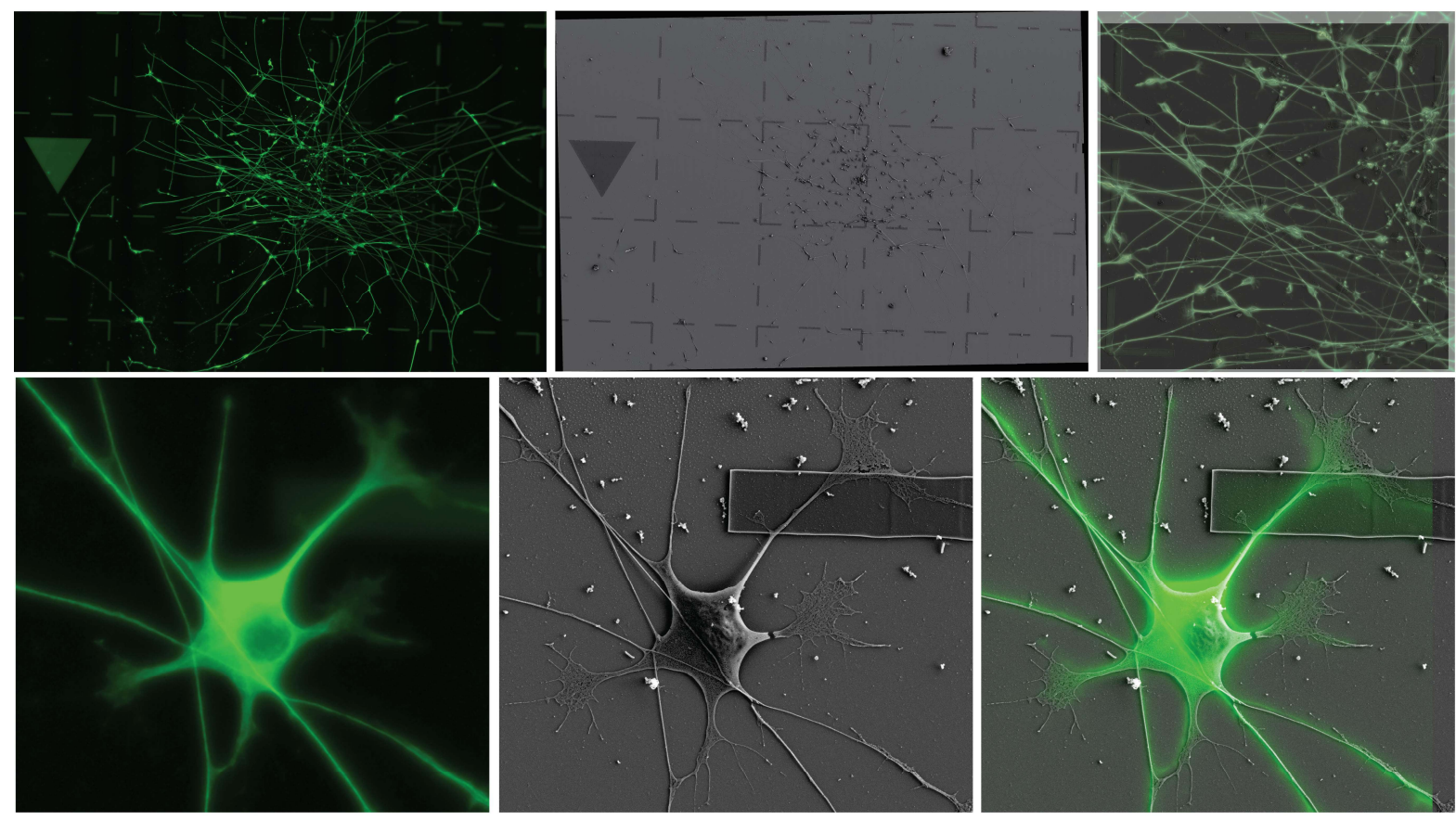

Figure 1. Fluorescent neurons labeled with Tuj1 and corresponding SEM image. Image overlay of area of interest. Lower Row: High-resolution image of area from above: fluorescence, SEM and overlay. One grid square is 500 microns. 